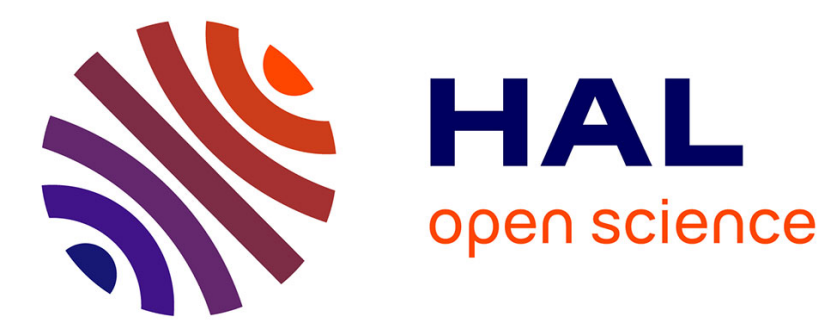

\title{
EFFETS DYNAMIQUES EN MÉCANIQUE DE LA RUPTURE FRAGILE DES INTERFACES
}

\author{
O. Coussy
}

\section{To cite this version:}

O. Coussy. EFFETS DYNAMIQUES EN MÉCANIQUE DE LA RUPTURE FRAGILE DES INTERFACES. Journal de Physique Colloques, 1985, 46 (C5), pp.C5-207-C5-212. 10.1051/jphyscol:1985526 . jpa-00224756

\section{HAL Id: jpa-00224756 https://hal.science/jpa-00224756}

Submitted on 1 Jan 1985

HAL is a multi-disciplinary open access archive for the deposit and dissemination of scientific research documents, whether they are published or not. The documents may come from teaching and research institutions in France or abroad, or from public or private research centers.
L'archive ouverte pluridisciplinaire HAL, est destinée au dépôt et à la diffusion de documents scientifiques de niveau recherche, publiés ou non, émanant des établissements d'enseignement et de recherche français ou étrangers, des laboratoires publics ou privés. 


\title{
EFFETS DYNAMIQUES EN MÉCANIQUE DE LA RUPTURE FRAGILE DES INTERFACES
}

\author{
0. Coussy \\ Laboratoire Central des Ponts et Chaussées, 58 boulevard Lefebvre, \\ 75732 Paris Cedex 15, France
}

\begin{abstract}
Résumé - On étudie ici la propagation dynamique d'une fissure à l'interface de deux demi-espaces infinis sous chargements plans ou antiplans dans le cadre du critère de Griffith. On donne tout d'abord l'extension naturelle de la formule d'Ir win générglisée pour les problèmes élastiques antiplans. On montre ensuite l'importance de l'effet de contact unilatéral pour les problèmes plans par rapport au problème d'un seul milieu et comment il faut modifier alors la formule d'Irwin. On étend enfin l'analyse aux milieux viscoélastiques.
\end{abstract}

\begin{abstract}
The dynamic propagation of an interface crack is studied under conditions of plane or antiplane loadings for the Griffith's criterion. We first give the generalized antiplane Irwin's formula for elastic problems. We then show the importance of the unilateral contact effect in plane interface problems. We show how to modify the Irwin's formula to take this effect into account. Finally we extend the analysis to viscoelastic problems.
\end{abstract}

\section{I - RESULTATS GENERAUX EN MECANIQUE DE LA RUPTURE FRAGILE DES INTERFACES}

Considérons une fissure semi infinie se propageant le long d'une interface de deux milieux semiinfinis $\left((a x e) 0 x_{1}\right)$ à une vitesse $V$, notée aussi $\ell$ ( $\ell$ pour longueur de fissure). Par la suite l'indice 1 ou 2 désignera respectivement le milieu supérieur ou inférieur. Il est bien connu $|1|$ que l'équilibre thermodynamique requiert que le flux d'énergie ou puissance disponible pour faire avancer la fissure est donné par :

$$
\mathcal{F}=\dot{W}_{e}-\dot{\Phi}_{R}-\dot{K}_{R}-\dot{D}_{R}
$$

où la notation pointée indique la dérivée temporelle de la quantité. Dans $(1), W_{e}$ est le travail des forces extérieures agissant sur la région matérielle $R$ entourant la tête de fissure, tandis que $\Phi_{R}$ est la dérivée temporelle de l'ẹnergie totale libre, la température étant maintenue constante. $K$ est l'énergie cinétique et $\dot{D}_{R}$ est la puissance dissipée volumique dans la région $R$. Le critère Griffith requiert que ce flux d'énergie est consommé dans la rupture de l'interface de telle manière que l'on ait :

$$
\mathcal{F}=2 \gamma V=2 \gamma \dot{\ell}
$$

où $\gamma$ est une énergie spécifique de rupture par unité de surface.(2) peut être formellement réécrit sous la forme :

$$
F=(F / V) ; F=2 \gamma
$$

où $\mathrm{F}$ a la dimension d'une force. Pour des propagations stationnaires, $\mathrm{K}_{\mathrm{R}}=0$ et $\mathrm{F}$ peut être identifié au taux de restitution de l'énergie $G$, défini par $G=-\partial U_{R} / \partial \ell_{\text {où }} U_{R}$ est le potentiel total pour la région $R|1|$. Ainsi pour $\dot{K}_{R}=0$, F est une force thermodynamique associée à $\ell$ par le potentiel $\mathrm{U}_{\mathrm{R}}$. Pour des propagations transitoires, ce n'est par contre pas le cas, en raison de l'énergie rayonnée par les ondes consécutives au démarrage dela fissure,et $\dot{K}_{R} \neq 0$ entraîne F $\neq$ GV. L'équation (1), qui met en jeu les champs de déplacement et de contraintes dans tout l'espace, n'est pas bien adaptée aux applications. Pour des problèmes antiplans, utilisant la mê-

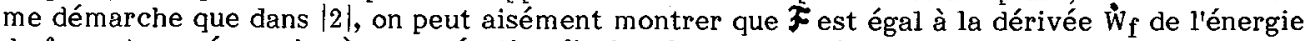
de fermeture nécessaire à une opération fictive de recollage inverse :

$$
d t \rightarrow 0: F=\dot{W}_{f}=\frac{1}{d t} \int_{v t}^{v t+v d t} \sigma_{23}\left(x_{1}\right) \Delta \mu\left(x_{1}-v d t\right) d x_{1}
$$

où $\Delta$ u et $\sigma_{23}$ sont respectivement l'ouverture de la fissure et la contrainte de cisaillement $\left(0 x_{3}\right.$ est la direction infinie du problème) derrière et devant la tête de fissure. 


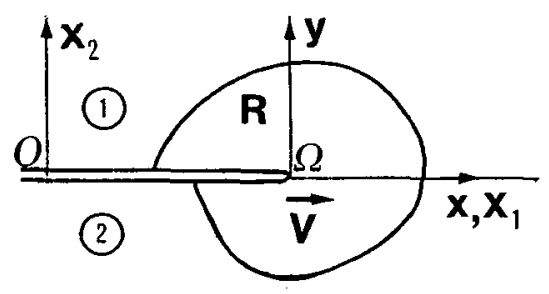

Fig. 1 - La géométrie du problème.

Pour obtenir (4) nous avons supposé un transport parallèle dans la direction de propagation $0 \mathrm{x}_{1}$ de ces champs asymptotiques. De fait la validité de cette hypothèse dépend des lois de comportement des matériaux. Pour des matériaux élastiques il est bien connu $|3|$ que le champ de contraintes en avant de la fissure est singulier en $\left(x_{1}-v t\right)^{-1 / 2}$ tandis que $\Delta u$ est proportionnel à $\left(\mathrm{Vt}-\mathrm{x}_{1}\right)^{1 / 2}$ derrière la pointe de fissure. On peut alors introduire le facteur d'intensité dynamique et les facteurs d'intensité cinématiques déjà introduits dans $|3|$ pour le problème d'un seul milieu, et qui seront ici définis par :

$$
\begin{aligned}
& k^{\sigma}=\operatorname{Lim} \sigma_{23} \sqrt{2 \pi}\left(x_{1}-v t\right)^{t / 2} ; x_{1} \rightarrow(v t)^{+}, x_{2}=0 \\
& K_{j}^{\mu \nu}=\operatorname{Lim} \frac{1}{2} \mu_{j}\left|\mu_{j}\right| \sqrt{2 \pi}\left(v t-x_{1}\right)^{-1 / 2} ; x_{1} \rightarrow(v t)_{1}, x_{2}=0
\end{aligned}
$$

où $\mu_{j}$ et $u_{j}$ sont respectivement le module de cisaillement et le champ de déplacement pour le milieu indicé par $j\left(u=u_{1}-u_{2}\right)$. Utilisant (5), (3) et (4) on obtient la formule d'Irwin généralisée :

$$
F=\frac{1}{2}\left\{\frac{1}{2 \mu_{1}} K_{1}^{\mu}+\frac{1}{2 \mu_{2}} K_{2}^{\mu}\right\} K^{\sigma}
$$

Ainsi les équations (3) et (6) montrent que seuls les facteurs d'intensité caractérisant les champs asymptotiques, sont à déterminer pour obtenir la loi de propagation d'une fissure à l'interface de deux milieu élastiques. En fait, seul le facteur d'intensité dynamique est réellement nécessaire, car on peut montrer $|4|$ que pour des milieux élastiques $\mathrm{K}^{\sigma}$ et $\mathrm{K}_{\mathrm{j}}$ vérifient la relation suivante, indépendante du chargement :

$$
K^{\sigma}=K_{j}^{\mu}\left(1-V^{2} / c_{j}^{2}\right)^{1 / 2}
$$

où $C_{j}$ est la vitesse des ondes de cisaillement.

\section{PROBLEMES ANTIPLANS ELASTIQUES}

Supposons à l'instant $\mathrm{t}=0 \mathrm{qu}^{\prime}$ une fissure commence à s'étendre à partir de l'origine des coordonnées dans les deux directions de $\mathrm{l}^{\prime} \mathrm{axe} 0 \mathrm{x}_{1}$ le long de l'interface de deux milieux à une vitesse $V$ telle que $V<C_{1}<C_{2}$.. Les contraintes à l'infini sont supposées nulles tandis que les faces de la fissure $\left(\left|x_{1}\right|<v t\right)$ sont soumises à un chargement antiplan d'intensité $\sigma_{23}^{\circ}$. Les ondes rayonnées sont décrites à la figure 2 . Quand la fissure commence à se propager, elle engendre une onde cylindrique dans chaque milieu, tandis que des ondes de tête, dues à l'excitation de l'interface par l'onde se propageant dans le milieu inférieur plus rapidement, sont engendrées en avant de l'onde cyli ndrique du milieu supérieur. Ces résultats peuvent être obtenus par des méthodes de résolution par fonctions autosemblables. Utilisant ces techniques, on peut obtenir $\mathrm{K} \sigma$ et montrer, par (3), (6) et (7), que le critère de propagation s'écrit $|4|$ :

$$
\begin{aligned}
& \left(\dot{w}_{f} / 2 \gamma v\right)\left(\Sigma^{2} l_{0} / \Sigma_{0}^{2} l\right) g(v)=1 \\
& \left(\dot{w}_{f} / 2 \gamma v\right)\left(\sigma^{2} l / \sigma_{0}^{2} l_{0}\right) f(v)=1
\end{aligned}
$$




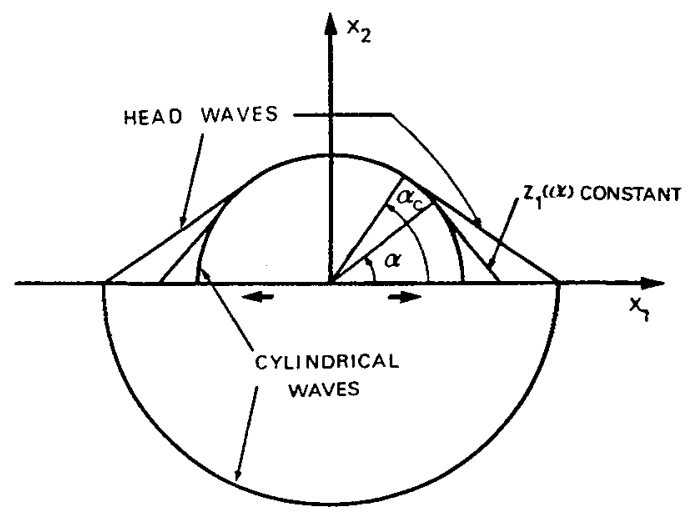

Fig. 2 - Ondes engendrées par la fissure.

L'équation ( $8 \mathrm{a}$ ) correspond à un chargement concentré $\Sigma$ appliqué à l'origine $\left(\sigma_{23}^{\circ}=\Sigma \delta\left(x_{1}\right) H(t)\right.$, $\delta$ et $\mathrm{H}$ fonctions delta et échelon), tandis que l'équation ( $8 \mathrm{~b}$ ) correspond à un chargement uniforme $\left(\sigma_{23}^{*}=\sigma\right)$, l'indice inférieur 0 désignant le chargement critique statique $\left(\mathrm{V} \rightarrow 0, \mathrm{Vt} \rightarrow \ell_{0}\right)$ et $\ell$ étant la longueur Vt. La figure 3 représente les variations de la fonction $g(V)$ pour différents matériaux, $f(V)$ ayant les mêmes variations.

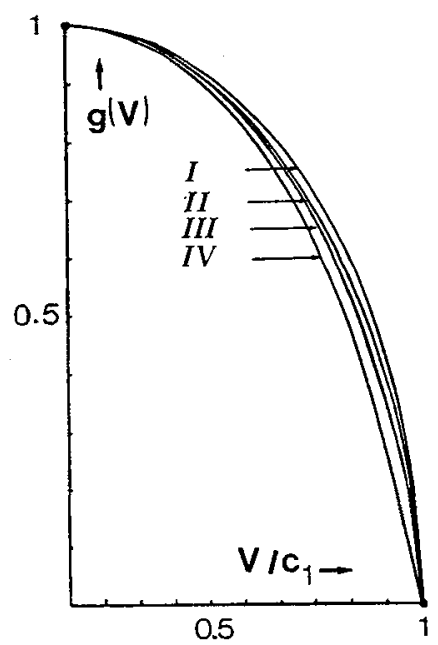

Fif. 3 - La fonction $g(v)$.

I mêmes matériaux ou élastique 1-rigide $2, \mathrm{II} \mu_{1} / \mu_{2}=0,054, \mathrm{C}_{1} / \mathrm{C}_{2}=0,3$ (epoxy 1 - verre 2 ), III $\mu_{1} / \mu_{2}=0,208, C_{1} / C_{2}=0,83$ (béton 1 , acier 2 ), IV $\mu_{1} / \mu_{2}=2,63, C_{1} / C_{2}=0,932$ (acier 1 aluminium 2).

Les fonctions $g(V)$ et $f(V)$ quantifient la perte en puissance disponible pour faire avancer la fissure dans le cas dynamique par rapport au cas statique, à longueur de fissure équivalente $\ell=\ell_{0}$. Durant la propagation, l'augmentation en énergie cinétique et la perte par rupture est obtenue par diminution de l'énergie libre totale et de l'énergie potentielle des efforts extérieurs (cf. (1), (3) pour $\dot{\mathrm{D}}_{\mathrm{R}}=0$ ). Les fonctions $\mathrm{f}$ et $\mathrm{g}$ valent 0 pour $\mathrm{V}=\mathrm{C}_{1}$, qui constitue ainsi une vitesse limite de propagation. Notons que (8a) implique en outre que la rupture peut être contrôlée pour un chargement concentré (i.e $\Sigma=$ constante $>\Sigma(\mathrm{V}) \mathrm{V} \rightarrow 0$ pour des temps $\mathrm{t}$ augmentant) contrairement au cas du chargement uniforme $\sigma$. 


\section{EFFETS DE CONTACT UNILATERAL POUR DES MOUVEMENTS PLANS STATIONNAIRES}

Retournons à la fissure semi infinie de la figure 1, mais considérons maintenant des problèmes plans correspondant à des chargements agissant dans les directions $\Omega$ x et $\Omega$ y du système de coordonnées attaché à la pointe de fissure et se déplaçant à une vitesse constante $V$. Pour des propagations stationnaires (pas d'instant initial et chargement fixe dans le système d'axe commobile $\Omega \mathrm{xy}$ ), on peut montrer $|5|$ que la solution plane présente un aspect non physique en tête de fissure. Il s'agit d'une violente oscillation de l'ouverture normale $\Delta v$ de la fissure suivant l'axe $\Omega y$, alternativement positive et négative et conduisant ainsi à une interpénétration des deux milieux. Dans $|5|$, il est stipulé que cette interpénétration peut être négligée dans le calcul de F car cette interpénétration n'a lieu que sur une zone extrêmement confinée juste derrière la tête de fissure. En fait, pour un problème statique, certains auteurs $|6|$, supposant qu'il y ait toujours un contact normal $(\Delta v=0)$ sur une courte longueur derrière la tête de fissure, ont montré que la rupture de l'interface ne peut intervenir que par une rupture en mode de cisaillement ou mode II. Sur la petite zone de contact, le champ de contraintes est un champ de compression, empêchant toute restitution en mode $\mathrm{I}$. Ce phénomène est dû à la différence des coefficients de Poisson $\nu_{j}$ des deux matériaux. Ainsi le chargement de rupture obtenu en négligeant l'interpénétration (comme en $|5|$ ) sera surestimé d'un facteur $\sqrt{8}$ (voir $|6|$ ). En outre, en $|7|$, il est montré numériquement que, très près de la tête de fissure, la solution prenant en compte une petite zone de contact, coïncide rapidement avec la solution analytique oscillante, telle que celle déterminée en $|5|$. On notera que ce résultat est en accord avec le principe de Saint-Venant. Enfin, dans $|7|$, il est montré que le facteur d'intensité en cisaillement, obtenu numériquement (avec une zone de contact) peut être obtenu en prenant le maximum du facteur oscillant existant dans la solution analytique du champ asymptotique de cisaillement.

Toutefois ces résultats statiques sont fondés sur l'hypothèse a priori que la zone de contact est continue. De fait on peut montrer que cela est vrai par une analyse dynamique $|8|$. Prenant le maximum du facteur oscillant intervenant dans la solution analytique dynamique, on peut tout d'abord déterminer les facteurs d'intensité dynamiques et cinématiques (respectivement $\overline{\mathrm{K}}_{\text {II }}^{\sigma}$ et $\overline{\mathrm{K}}_{\mathrm{II}}^{\mathrm{u}} \mathrm{j}$ correspondant au champ de cisaillement singulier $\bar{\sigma}_{\mathrm{xy}}$ et l'ouverture tangentielle

$\Delta \vec{u}=\vec{u}_{1}-\vec{u}_{2}$ dans la direction $\Omega x$ ). Alors une estimation de $F$, pour une vitesse $V$ donnée, peut être :

$$
F(v)=\sum_{j=1,2} \frac{1-\nu_{j}^{2}}{2 E_{j}} \bar{K}_{I I}^{\mu_{j}} K_{I I}^{\sigma}
$$

où $E_{j}$ est le module d'Young. En fait, pour une vitesse extrêmement spéciale Vo, il peut être montré que la solution analytique ne présente pas d'oscillations et ainsi est la solution exacte. Dans ce cas, on peut déterminer l'expression exacte du flux d'énergie $\mathcal{F}$ (Vo) $=$ Fo Vo avec :

$$
F_{0}=\sum_{j=1,2} \frac{1-\nu_{j}^{2}}{2 E_{j}}\left(K_{I I}^{\mu_{j}} K_{I I}^{\sigma}+K_{I}^{v_{j}} K_{I}^{\sigma}\right), V=V_{0}
$$

où les facteurs d'intensité sont définis comme pour le milieu homogène $|3|$. Le fait intéressant est que la valeur $F(V o)$ donnée par l'estimation (9), est en fait la valeur exacte $F_{o}$ donnée par (10). Ceci veut dire que, bien qu'il y ait une discontinuité dans le mode de rupture de l'interface du passage de Vo ( $\mathrm{V} \neq \mathrm{Vo}$ mode II, $\mathrm{V}=\mathrm{Vo}$ mode I, pour un chargement de traction), il y a continuité du flux d'énergie au passage de Vo ce qui semble, pour le moins, conforme à la nature physique du phénomène. Ce résultat conduit à une forte présomption que la zone de contact est continue et que prendre le maximum du facteur oscillant correspond de fait à la solution exacte.

\section{EFFFETS VISCOELATIQUES EN MOUVEMENTS ANTIPLANS}

Dans cette partie le milieu inférieur est élastique tandis que le milieu supérieur est maintenant viscoélastique, obéissant à la loi de comportement :

$$
\left(\frac{d}{d t}+b\right)^{2} \sigma_{\alpha 3}=\mu_{0}\left(\frac{d}{d t}+a\right)^{2} \frac{\partial \mu}{\partial x_{\alpha}} ; \alpha=1,2 ;\left\{\begin{array}{l}
a / b=c_{\infty} / c_{0} \\
a=\delta / \tau, 0<\delta<1
\end{array}\right.
$$

Dans l'équation (11) $\mu_{0}$ est le module de cisaillement instantané, correspondant à la vitesse des ondes instantanée $C_{0}$ ou correspondant aux hautes fréquences, tandis que $C_{\infty}$ est la vitesse des ondes différée ou correspondant aux basses fréquences. $\tau$ est un temps de relaxation. Ce modèle a été étudié dans $|9|$ et conduit à des courbes de fluages peu différentes de celles du modèle linéaire standard. Supposons maintenant qu'à l'instant initial $t=0$, une fissure semi-infinie 
apparaît soudainement et commence à se propager avec une vitesse constante $\mathrm{V}$ sous l'action d'un chargement uniforme antiplan agissant sur les faces de la fissure. Par une technique dite de Wiener-Hopf utilisée en $|\mathbf{1 0}|$, on peut montrer que les solutions pour des temps petits et grands vérifient (Coussy, à paraître) :

$$
K^{\sigma}=(2 i \pi) \sqrt{2} \sigma \sqrt{(C-v) t} \text { exp } \Lambda_{C}, V<C
$$

où $\mathrm{C}=\mathrm{C}_{0}$ ou $\mathrm{C}_{\infty}$ pour $\mathrm{t} \rightarrow{ }_{\infty}^{\circ}$ respectivement, le terme exponentiel correspondant aux effets des ondes de tête en avant des ondes rayonnées par la fissure et décrites à la figure 4. Les mêmes commentaires peuvent être faits que dans le cas de la figure 2. La différence essentielle est que, au fur et à mesure que les ondes se propagent,elles perdent progressivement leurs composantes hautes fréquences et progressivement la vitesse des ondes décroît de $\mathrm{C}_{\mathrm{o}}$ à $\mathrm{C}_{\infty}$ lorsque le temps augmente.

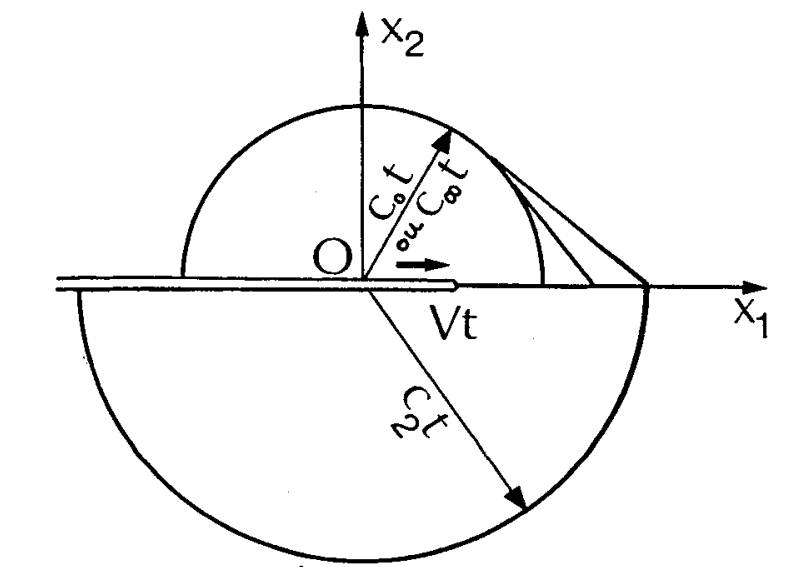

Fig. 4 - Ondes rayonnées par la fissure pour $\mathrm{V}<\mathrm{C}_{0}$ ou $\mathrm{C}_{\infty},(\mathrm{t} \rightarrow \mathrm{O}$ ou $\mathrm{t} \rightarrow \infty)$.

En outre (7) est toujours vérifié mais avec $\mathrm{C}_{\mathrm{j}}=\mathrm{C}_{\mathrm{O}}$, ceci même dans la solution à long terme $(t \rightarrow \infty)$. Ainsi le phénomène est contrôlé par la relaxation du champ de contraintes en tête de fissure, qui induit derrière cette tête un champ de déplacement, correspondant aux caractéristiques instantanées $\mu_{0}$ et $\mathrm{C}_{\mathrm{O}}$, en raison de la perturbation instantanée engendrée par la propagation de la fissure.

La différence essentielle entre le problème d'interface et le problème homogène (la loi de comportement (11) est valable dans tout l'espace) est que le champ de contraintes est singulier en $\mathrm{x}^{-\frac{1}{2}}$ pour tous les temps dans le cas homogène $|10|$ alors que cela n'est vrai qu'asymptotiquement $(t \rightarrow 0$ ou $t \rightarrow \infty$ ) pour le cas de l'interface . Dans ce dernier cas, pour des temps petits, le processus de relaxation n'a pas encore commencé, tandis que pour des temps grands il est complètement achevé. Pour des temps intermédiaires ce processus de relaxation est gêné par le comportement élastique du milieu inférieur, le champ de contraintes en tête de fissure devenant difficile d'analyse.

L'équation (12), pour des temps grands, n'est valable toutefois que pour $\mathrm{V}<\mathrm{C}_{\infty}$. Une autre solution existe pour $\mathrm{V}>\mathrm{C}_{\infty}$ et $\mathrm{t} \rightarrow \infty$ :

$$
\begin{aligned}
& V>C_{\infty} \text { et } t \rightarrow \infty: \\
& K^{\sigma}=\sigma \sqrt{2} \exp \wedge\left(v \tau c_{0}\left(c_{0}-v\right) / c_{\infty}\left(v-c_{\infty}\right)\right)^{-1 / 2}
\end{aligned}
$$

Cette dernière solution est une solution stationnaire, contrairement à la solution transitoire (12). Utilisant (3) avec $\boldsymbol{\mu}_{1}=\boldsymbol{H}_{0},(7)$, (12) et (13), on peut alors montrer que pour des chargements suffisamment grands par rapport à $2 \gamma$, la vitesse de propagation de la fissure peut dépasser $\mathrm{C}_{\infty}$, avant que le processus de relaxation, gouverné par le temps $\tau$, ne soit achevé et que la vitesse des ondes dans le milieu supérieur ne soit effectivement $C_{\infty}$. Dans ce cas, la vitesse terminale de la fissure n'est ni $\mathrm{C}_{0}$ ou $\mathrm{C}_{\infty}$, mais une vitesse intermédiaire donnée par les équations cidessus mentionnées. Pour conclure, notons que (12) ne fait intervenir que $\mathrm{C}_{O}$ ou $\mathrm{C}_{\infty}$ et semble ainsi général, tandis que (13), faisant intervenir un temps de relaxation lié au modèle choisi, doit de fait dépendre du modèle viscoélastique retenu. 


\section{REFERENCES}

11 NGUYEN Q.S., J.M.T.A. 19 (1980) 363.

2) ERDCGAN F., Fracture 2, Liebowitz editor (1968) 497.

$3 \mid$ BUI H.D., Mécanique de Ia rupture fragile, Masson (1978).

4 COUSSY O., C.R.A.S. Paris, 299 (1984) 1101.

$5 \mid$ WILLIS J.R., J. Mech. Phys. Solids 19 (1971) 353.

6 WANG S.S.CHAO I., Jnl. of Appl. Mech. 50 (1983) 169.

7| COMMINOU. M., Jnl. of Appl. Mech. 44 (1977) 631.

$8 \mid$ COUSSY O., J.M.T.A., accepté pour publication.

9| ACHENBACH J.D., CHAQ C., C., J. Mech. Phys. Solids 10 (1962) 245.

$|10|$ ATKINSON C., LIST, R.D., Int. J. Engng. Sci. 10 (1972) $\overline{30}$. 